\title{
Television viewing, computer game play and book reading during meals are predictors of meal skipping in a cross- sectional sample of 12-, 14- and 16-year-olds
}

\author{
Kathleen Custers* and Jan Van den Bulck \\ Leuven School for Mass Communication Research and Leuven Food Science and Nutrition Research Centre \\ (LFoRCe), Katholieke Universiteit Leuven, Parkstraat 45, Box 3603, B-3000 Leuven, Belgium
}

Submitted 25 February 2009: Accepted 27 July 2009: First published online 22 September 2009

\begin{abstract}
Objective: To examine whether television viewing, computer game playing or book reading during meals predicts meal skipping with the aim of watching television, playing computer games or reading books (media meal skipping). Design: A cross-sectional study was conducted using a standardized selfadministered questionnaire. Analyses were controlled for age, gender and BMI. Setting: Data were obtained from a random sample of adolescents in Flanders, Belgium.

Subjects: Seven hundred and ten participants aged 12, 14 and 16 years.

Results: Of the participants, $11 \cdot 8 \%$ skipped meals to watch television, $10 \cdot 5 \%$ skipped meals to play computer games and $8 \cdot 2 \%$ skipped meals to read books. Compared with those who did not use these media during meals, the risk of skipping meals in order to watch television was significantly higher for those children who watched television during meals $(2 \cdot 9$ times higher in those who watched television during at least one meal a day). The risk of skipping meals for computer game playing was 9.5 times higher in those who played computer games weekly or more while eating, and the risk of meal skipping in order to read books was 22.9 times higher in those who read books during meals less than weekly. The more meals the respondents ate with the entire family, the less likely they were to skip meals to watch television.

Conclusions: The use of media during meals predicts meal skipping for using that same medium. Family meals appear to be inversely related to meal skipping for television viewing.
\end{abstract}

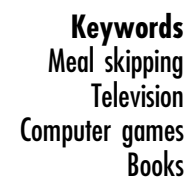

Meal skipping has been identified as a potential threat to healthy eating habits ${ }^{(1,2)}$. Although research about this topic is scarce, a study in 2006 showed that skipping meals may lead to increased levels of fat mass in adolescents ${ }^{(3)}$. Some studies found an association between meal frequency and obesity in children and the elderly. A questionnaire on risk factors for obesity, completed by the parents of 4370 German children, showed that increasing meal frequency may reduce the prevalence of obesity ${ }^{(4)}$. Dubois and colleagues found that pre-school children who skipped breakfast had a higher BMI as a result of an increased intake of energy and carbohydrates ${ }^{(5)}$.

If meal skipping is a threat to healthy eating habits, it is important to study the reasons people may have for such behaviour. A survey of 2546 adolescents suggested that media use was one of the reasons why adolescents skipped meals. At least one in ten adolescents skipped at least one meal a week in order to watch television or to play computer games. Meal skipping for television viewing occurred more regularly in adolescents who watched more television and in those who played computer games more often ${ }^{(6)}$. Such behaviour is referred to as 'media meal skipping' in the remainder of the present paper.

Media meal skipping should be distinguished from having a meal while using media. At least in theory, there is no need for an adolescent to skip a meal in order to watch television if the television is on during meals. It has, however, been shown that having the television set on during meals is associated with unhealthy eating patterns $^{(7)}$ and more frequent snacking ${ }^{(8)}$. Feldman and colleagues found that adolescents who watched television during family meals consumed fewer vegetables, less Ca-rich food and grains, and more soft drinks. Nevertheless, adolescents who watched television during family meals had a more healthy diet than adolescents who did not consume regular family meals ${ }^{(9)}$. It has been shown that television viewing during meals is also related to increased meal frequency. When participants indicated 
that they watched television while eating on a particular day, they also ate more frequently on that particular day ${ }^{(10)}$. Two processes thus appear to exist: on the one hand, media use during meals appears to be associated with less meal skipping; on the other, media use may often be a reason for more meal skipping. We wanted to examine the relationship between these two processes: whether media use during meals was associated with more or less media meal skipping. Media use during meals might protect against media meal skipping because it enables media use without having to forego meals. Alternatively, it may also be an indicator of less stringent family rules regarding media use and meals, in which case it might be an indicator of more media meal skipping.

Television is not the only medium that may be of concern. A previous study on media use and meal skipping looked at both television viewing and computer games $^{(6)}$. Another study showed that book reading did not behave like television viewing or computer game play with regard to health-related effects ${ }^{(11)}$.

While media use may encourage meal skipping, communal family meals appear to prevent this behaviour. Studies examining the association between family meal patterns and eating habits of adolescents concluded that family meals appeared to be an important catalyst of the intake of healthy foodstuffs in adolescents ${ }^{(12-14)}$. Positive associations were found between frequency of family meals and the intake of fruits, vegetables, grains and $\mathrm{Ca}-$ rich foods. In addition, frequency of family meals was negatively associated with the consumption of soft drinks. Larson and colleagues also looked at the association between frequency of family meals and overall meal frequency. The authors found that frequency of family meals was associated with more breakfast meals in females. In addition, it was associated with an increase in the frequency of dinner in both sexes ${ }^{(13)}$.

The aims of the present study were therefore to examine: (i) whether using media during regular meals predicts meal skipping to use that same medium; and (ii) whether eating meals together with the family is negatively related to meal skipping. The study considered three types of media: television, computer games and book reading.

\section{Methods}

\section{Participants}

Respondents were selected from a random sample of seventh, ninth and eleventh grade students in four secondary schools in Flanders (the Dutch-speaking part of Belgium). Data were collected in November 2007. A twostep sampling method was used. First, schools were selected randomly from the official list of secondary schools of the Department of Education in Flanders. If a school was willing to cooperate, all students in the seventh, ninth and eleventh grade in that school were selected for inclusion in the sample. The study was presented as an omnibus study on the leisure habits of Flemish youngsters and took place in an assembly setting. A numbering system was used so that anonymity could be guaranteed to the respondents. Informed consent was obtained from the legal guardians of the children. The study was reviewed and approved by the authors' Institutional Review Board.

The sample size of the study was 710 (response rate $89 \%$ ). Of the sample, $45 \cdot 1 \%$ were boys. Of the participants, $45 \cdot 5 \%$ were seventh grade students, $27 \cdot 2 \%$ were ninth grade students and $27 \cdot 3 \%$ were eleventh grade students. The average age was $13 \cdot 70$ (SD 1.74) years.

\section{Measures}

The study used data collected by means of a standardized, self-administered questionnaire measuring the frequency of general meal skipping, media meal skipping, family meals and media use during meals. Meal frequency questionnaires often ask respondents to report their eating behaviours of the seven preceding days. Because media meal skipping may not occur weekly, this would have led to a considerable underestimation of the prevalence of these behaviours. We therefore opted to use answer categories referring to the frequency of those behaviours in the past year (as done in a previous study on media meal skipping) ${ }^{(6)}$.

\section{Meal skipping}

General meal skipping was measured by asking respondents how often they skipped breakfast, lunch and dinner on a yearly basis in three separate questions. What may look like an ordinal scale by the way it is measured is often actually a continuous latent variable (measured with error at the manifest level) ${ }^{(15)}$. We therefore recoded the response categories into approximations of the number of days per year each category corresponded to.

Response items (and their corresponding estimated number of days per year) were: $1=$ never $(0) ; 2=$ once a year (1); $3=$ once a month (12); $4=$ several times a month (36); $5=$ once a week (52); $6=$ several times a week (156); 7 = every day (365). By summing these three variables we obtained an estimate of the number of meals skipped per year.

\section{Media meal skipping}

Van den Bulck and Eggermont ${ }^{(6)}$ asked their respondents how often they skipped 'a meal' to use a specific medium. To measure media meal skipping more accurately in the present study and to make the mental arithmetic easier for the respondents, we listed breakfast, lunch and dinner separately. Three questions were therefore asked for television viewing, computer games playing and reading books, creating nine variables. Respondents were asked: 'How often do you skip breakfast/lunch/dinner in order 
to watch television/play computer games/read books?' Response items were the same as for general meal skipping and were recoded the same way. A large percentage of respondents did not report any media meal skipping. The dependent variables were therefore dichotomized so that ' 0 ' meant that this particular type of behaviour was never reported and ' 1 ' meant it had been reported at least once.

\section{Media use while consuming meals}

The respondents were queried how often they watched television while having breakfast, lunch or dinner. The same questions were asked regarding playing computer games and reading books, thus creating nine variables. Response items were the same as for meal skipping.

\section{Frequency of family meals}

To measure the frequency of family meals, the respondents were queried, in three separate questions, how often they ate breakfast, lunch and dinner together with their family. Response items were the same as for meal skipping.

\section{Control variables}

We controlled for gender and age group. Participants with a higher weight for mass ratio might have different eating behaviours and both television viewing and family meals have been shown to be associated with body mass ${ }^{(16)}$. We therefore asked participants to report their weight and height in order to compute the BMI and we adjusted for this variable.

\section{Statistical analysis}

All statistical analyses were conducted using the SPSS $15^{\mathrm{TM}}$ for Windows statistical software package (SPSS Inc., Chicago, IL, USA). The variables measuring eating behaviours referred to the number of days on which these behaviours occurred. While the underlying variable was continuous, bias was minimized by treating them as rank order variables. Differences between boys and girls were therefore tested with the Mann-Whitney $U$ test for independent samples and the relationship with BMI and age was estimated using Spearman rank correlations. Three logistic regression analyses were run to assess the relationship between using a particular type of media while eating and skipping meals because of that same medium. For reading books and playing computer games while eating, the number of participants who reported indulging in this kind of behaviour on a daily basis was so small that they were added to the preceding category ('weekly or more'). The frequency of eating meals together as a family was entered as a potentially protective variable. Gender, age and BMI were added as control variables, but are reported only when they were statistically significant.

\section{Results}

\section{Prevalence of meal skipping}

Overall, $53 \cdot 4 \%$ of the participants said they had skipped meals. In those who had skipped meals an estimated average of $91 \cdot 1$ (SD 135.7) meals per year were skipped. Spearman rank order correlations showed that meal skipping correlated significantly with media meal skipping for television $(\rho=0.30, P<0.0001)$, computer games $(\rho=0 \cdot 20, P<0 \cdot 0001)$ and book reading $(\rho=0 \cdot 14$, $P<0 \cdot 0001)$.

\section{Prevalence of media meal skipping}

Almost $12 \%$ of the participants reported they skipped meals to watch television at least occasionally. There was no statistically significant difference between boys and girls or between age groups. There was no significant difference between those who skipped meals for television and those who did not, as regards their BMI.

Playing computer games was reported as a reason for skipping meals in $10.5 \%$ of the participants. Boys were 2.5 times more likely than girls to skip meals for this reason $(95 \%$ CI $1.55,3.92)$, but neither age group nor BMI differed between skippers and non-skippers.

Reading books was reported as a reason for skipping meals in $8 \cdot 2 \%$ of the participants. There were no significant differences between genders and age groups or according to BMI.

\section{Prevalence of media use while consuming meals}

Television viewing while eating was the most prevalent of the three media meal behaviours. Of the respondents, $37.7 \%$ never watched television while eating, 18.9\% watched television while eating less than once a week, $25.5 \%$ did it at least once a week and a further $17.9 \%$ did it for at least one meal a day. There was no difference between boys and girls, but eating with the television switched on increased very slightly with age (Spearman's $\rho=0 \cdot 09, P=0 \cdot 02$ ).

Of the respondents, $88 \cdot 8 \%$ reported never to play games while eating, $6 \cdot 7 \%$ did it less than weekly, $3 \cdot 7 \%$ did it at least weekly and $0.7 \%$ claimed they did it daily. Some $75.4 \%$ of the respondents reported never to read books while eating a meal, $15 \cdot 5 \%$ reported doing it occasionally but less than once a week, $7 \cdot 7 \%$ did it at least once a week and $1 \cdot 4 \%$ did it daily.

\section{Frequency of family meals}

For $12.9 \%$ of the participants all meals were family meals. A further $20.7 \%$ claimed that only one meal a year was not a family meal and $20 \cdot 8 \%$ reported that a meal without the entire family occurred less than once a month. Some $27 \cdot 8 \%$ did not have family meals once a month or more and $18 \cdot 0 \%$ had at least one meal per day without the rest of the family.

Girls were more likely not to have family meals (Spearman's $\rho=0 \cdot 11, P=0 \cdot 004$ ). Not eating with the 
family also increased with age (Spearman's $\rho=0 \cdot 22$, $P<0 \cdot 0001$ ) and BMI (Spearman's $\rho=0 \cdot 25, P<0 \cdot 0001$ ).

\section{Media meal skipping}

Meal skipping for watching television

The logistic regression analysis in Table 1 shows that the fewer meals the participants had together with the family, the more likely they were to skip meals to watch television. Compared with the reference category, which always ate together, skipping just one family meal a year did not appear to make a statistically significant difference (even though the odds doubled), but skipping just a few led to a sixfold increase in the odds of skipping a meal for television viewing. In those who did not have a family meal at least once a week the odds increased eightfold, and in those who did not have a family meal at least once a day the odds were five times higher.

In those who never watched television while eating, $7 \cdot 8 \%$ claimed to skip meals for television viewing. Watching television while eating less than once a week did not appear to affect the odds of skipping a meal in order to watch television, but those who watched television while eating at least once a week were $2 \cdot 2$ times more likely also to skip meals to watch television and those who watched television during at least one meal a day were $2 \cdot 9$ times more likely to skip meals for television.

\section{Meal skipping for playing computer games}

While those who ate all meals or nearly all meals together had the lowest percentage of participants reporting meal skipping for playing computer games, the odds of skipping meals to play computer games were not significantly different for lower levels of participation in family eating.

Those who played computer games while eating were much more likely to skip meals to play computer games.
Among those who never played computer games while eating, only $6 \cdot 5 \%$ skipped meals to play computer games. Among those who played games while eating occasionally the odds were more than sevenfold those of the reference category. In that group $37 \cdot 0 \%$ of the respondents skipped meals for playing computer games. The odds rose to $9 \cdot 5$ times in the group which played games while eating at least weekly. In that group over half $(51 \cdot 7 \%)$ skipped meals to play computer games (see Table 2).

\section{Skipping meals to read books}

There were no significant differences between the frequency with which participants took part in family meals and the extent to which they reported skipping meals to read books.

Those who never read books while eating were least likely to skip meals to read books. Those who did read while eating, even occasionally, were a lot more likely to skip meals for reading books. In the group which read while eating less than weekly, 22.9\% reported having skipped meals in order to read (more than a sevenfold increase in odds). Among those reading weekly or more, $14.5 \%$ had skipped meals for reading books (see Table 3 ).

\section{Discussion}

There are many reasons to regard meal skipping as a potential health threat. It has been linked to increased fat mass $^{(1,2)}$ and obesity ${ }^{(4)}$. A recent study identified media use as one particular reason for which adolescents tend to skip meals ${ }^{(6)}$. Of those participants who reported having skipped meals in the current study, 29.4\% also reported having skipped meals to watch television, playing computer games or reading books. Media meal skipping thus appears to be an important reason for meal skipping in adolescents. Our study looked at two potential predictors

Table 1 Logistic regression analysis for the relationship between eating while television viewing, eating family meals together and meal skipping in order to watch television: random sample of adolescents aged 12, 14 and 16 years in Flanders, Belgium

\begin{tabular}{|c|c|c|c|c|c|c|}
\hline Variable & $\begin{array}{l}\% \text { skips a meal to } \\
\text { watch television }\end{array}$ & $\begin{array}{l}\text { Regression } \\
\text { coefficient }\end{array}$ & SE & OR & $95 \% \mathrm{Cl}$ & $P$ value \\
\hline \multicolumn{7}{|l|}{ Eating family meals together } \\
\hline All meals together & $2 \cdot 3$ & - & - & $1 \cdot 00$ & ref & 0.040 \\
\hline One meal per year not with family & $4 \cdot 7$ & $0 \cdot 77$ & 0.83 & $2 \cdot 16$ & $0.42,11.06$ & 0.355 \\
\hline $\begin{array}{l}\text { Less than one meal per month not } \\
\text { with family }\end{array}$ & $15 \cdot 8$ & $1 \cdot 87$ & $0 \cdot 76$ & $6 \cdot 46$ & $1 \cdot 45,28 \cdot 83$ & $0 \cdot 014$ \\
\hline $\begin{array}{l}\text { Less than one meal per week not } \\
\text { with family }\end{array}$ & $15 \cdot 9$ & $1 \cdot 87$ & $0 \cdot 76$ & $6 \cdot 46$ & $1 \cdot 45,28 \cdot 66$ & $0 \cdot 014$ \\
\hline $\begin{array}{l}\text { One meal per week or more not } \\
\text { with family }\end{array}$ & $19 \cdot 2$ & $2 \cdot 09$ & $0 \cdot 91$ & $8 \cdot 12$ & $1 \cdot 35,48 \cdot 67$ & 0.022 \\
\hline At least one meal a day not with family & $15 \cdot 7$ & $1 \cdot 69$ & $0 \cdot 78$ & $5 \cdot 42$ & $1 \cdot 17,25 \cdot 14$ & 0.031 \\
\hline \multicolumn{7}{|l|}{ Television is on while eating } \\
\hline Less than weekly & $9 \cdot 4$ & 0.47 & 0.41 & 1.60 & $0 \cdot 71,3 \cdot 61$ & 0.257 \\
\hline Weekly or more & $14 \cdot 6$ & $0 \cdot 80$ & 0.35 & $2 \cdot 23$ & $1 \cdot 11,4 \cdot 48$ & 0.024 \\
\hline At least for one meal per day & $20 \cdot 0$ & 1.06 & 0.39 & $2 \cdot 88$ & $1 \cdot 34,6 \cdot 18$ & 0.007 \\
\hline Model constant & - & $-5 \cdot 78$ & $2 \cdot 16$ & 0.01 & - & 0.008 \\
\hline
\end{tabular}

ref, referent category. 
Table 2 Logistic regression analysis for the relationship between eating while playing computer games, eating family meals together and meal skipping for computer games: random sample of adolescents aged 12, 14 and 16 years in Flanders, Belgium

\begin{tabular}{|c|c|c|c|c|c|c|}
\hline Variable & $\begin{array}{l}\% \text { skips a meal to play } \\
\text { computer games }\end{array}$ & $\begin{array}{l}\text { Regression } \\
\text { coefficient }\end{array}$ & SE & OR & $95 \% \mathrm{Cl}$ & $P$ value \\
\hline Boys & $15 \cdot 6$ & - & - & $1 \cdot 00$ & ref & - \\
\hline Girls & $6 \cdot 3$ & -0.73 & $0 \cdot 29$ & 0.48 & $0 \cdot 27,0.84$ & 0.012 \\
\hline \multicolumn{7}{|l|}{ Eating family meals together } \\
\hline All meals together & $7 \cdot 0$ & - & - & $1 \cdot 00$ & ref & $0 \cdot 182$ \\
\hline One meal per year not with family & $4 \cdot 3$ & -0.55 & $0 \cdot 62$ & 0.58 & $0 \cdot 17,1.95$ & 0.424 \\
\hline $\begin{array}{l}\text { Less than one meal per month not } \\
\text { with family }\end{array}$ & $15 \cdot 4$ & $0 \cdot 83$ & 0.52 & $2 \cdot 29$ & $0 \cdot 83,6 \cdot 29$ & $0 \cdot 130$ \\
\hline $\begin{array}{l}\text { Less than one meal per week not } \\
\text { with family }\end{array}$ & $11 \cdot 7$ & $0 \cdot 45$ & $0 \cdot 52$ & $1 \cdot 58$ & $0 \cdot 57,4 \cdot 39$ & $0 \cdot 447$ \\
\hline $\begin{array}{l}\text { One meal per week or more not } \\
\text { with family }\end{array}$ & $14 \cdot 8$ & $0 \cdot 69$ & $0 \cdot 75$ & 1.99 & $0 \cdot 46,8 \cdot 55$ & $0 \cdot 328$ \\
\hline At least one meal a day not with family & $13 \cdot 0$ & 0.54 & 0.54 & $1 \cdot 72$ & $0.59,4.97$ & 0.383 \\
\hline \multicolumn{7}{|l|}{ Playing computer games while eating } \\
\hline Never & $6 \cdot 5$ & - & - & $1 \cdot 00$ & ref & 0.000 \\
\hline Less than weekly & $37 \cdot 0$ & $2 \cdot 01$ & $0 \cdot 37$ & $7 \cdot 46$ & $3 \cdot 65,15 \cdot 27$ & 0.000 \\
\hline Weekly or more & $51 \cdot 7$ & $2 \cdot 25$ & 0.45 & $9 \cdot 50$ & $3.93,22.98$ & 0.000 \\
\hline Model constant & - & $-2 \cdot 60$ & 0.46 & 0.07 & - & 0.355 \\
\hline
\end{tabular}

ref, referent category.

Table 3 Logistic regression analysis for the relationship between eating while reading books, eating family meals together and meal skipping in order to read books: random sample of adolescents aged 12, 14 and 16 years in Flanders, Belgium

\begin{tabular}{|c|c|c|c|c|c|c|}
\hline Variable & $\begin{array}{l}\% \text { skips a meal to read } \\
\text { books }\end{array}$ & $\begin{array}{l}\text { Regression } \\
\text { coefficient }\end{array}$ & SE & OR & $95 \% \mathrm{Cl}$ & $P$ value \\
\hline \multicolumn{7}{|l|}{ Eating family meals together } \\
\hline All meals together & $5 \cdot 8$ & - & - & 1.00 & ref & $0 \cdot 228$ \\
\hline One meal per year not with family & $8 \cdot 2$ & $0 \cdot 24$ & 0.58 & $1 \cdot 27$ & $0.41,3.93$ & 0.683 \\
\hline $\begin{array}{l}\text { Less than one meal per month not } \\
\text { with family }\end{array}$ & $8 \cdot 1$ & $-0 \cdot 17$ & 0.61 & 0.84 & $0 \cdot 26,2 \cdot 77$ & 0.775 \\
\hline $\begin{array}{l}\text { Less than one meal per week not } \\
\text { with family }\end{array}$ & $12 \cdot 7$ & $0 \cdot 68$ & 0.54 & $1 \cdot 97$ & $0 \cdot 68,5 \cdot 70$ & $0 \cdot 210$ \\
\hline $\begin{array}{l}\text { One meal per week or more not } \\
\text { with family }\end{array}$ & $11 \cdot 5$ & $0 \cdot 64$ & $0 \cdot 80$ & $1 \cdot 89$ & $0.39,9 \cdot 06$ & $0 \cdot 427$ \\
\hline At least one meal a day not with family & $5 \cdot 2$ & $-0 \cdot 41$ & 0.64 & 0.67 & $0 \cdot 19,2 \cdot 35$ & 0.527 \\
\hline \multicolumn{7}{|l|}{ Reading books while eating } \\
\hline Never & $3 \cdot 9$ & _ & _ & 1.00 & ref & 0.000 \\
\hline Less than weekly & $22 \cdot 9$ & $2 \cdot 03$ & 0.33 & $7 \cdot 61$ & $3 \cdot 97,14 \cdot 60$ & 0.000 \\
\hline Weekly or more & $14 \cdot 5$ & $1 \cdot 32$ & 0.45 & 3.76 & $1.55,9.09$ & 0.003 \\
\hline Model constant & - & $-3 \cdot 34$ & 0.50 & 0.04 & - & 0.000 \\
\hline
\end{tabular}

ref, referent category.

of media meal skipping. It hypothesised that using media during meals might be a predictor of increased media meal skipping and that the practice of eating together with family members (family eating) might be a predictor of reduced media meal skipping. The results showed that meals were regularly accompanied by watching television, reading books and even playing computer games. Television viewing during meals was most prevalent. Use of media during meals was associated with more meal skipping to consume the same medium. The use of television while eating increased the odds of meal skipping to watch television. Playing computer games while eating made skipping meals to play computer games much more likely and reading while eating made skipping meals to read books more likely. Family meals (eating together with the family) made skipping meals to watch television less likely, but family meals were not associated with higher or lower levels of meal skipping for computer game play or book reading.

The current study thus shows that: (i) an important reason for skipping meals in adolescence is media use; (ii) using a particular type of media while eating is a good predictor of media meal skipping; and (iii) eating with the family is inversely related to media meal skipping, at least in the case of television viewing.

This study had limitations. First, the four schools which agreed to take part were geared towards preparing their students for higher education. This explains why meal skipping was less prevalent in the current study than in that by Van den Bulck and Eggermont ${ }^{(6)}$ which drew from a much broader student base. Previous research has shown that socio-economic status is an important 
predictor of eating behaviour: high socio-economic status is related to healthy food intake ${ }^{(17)}$ and less breakfast skipping in schoolchildren ${ }^{(18)}$. Moreover, lower socioeconomic status is associated with more television viewing while eating and a higher intake of unhealthy foodstuffs in adolescents ${ }^{(19)}$.

The second limitation is the cross-sectional design of the study. Cross-sectional studies are only able to identify associations. The fact that a dose-response relationship appeared to exist does point in the direction of causality, but other designs are needed to establish whether this was the case.

The third limitation is that this study relied on selfreports. Eating behaviour in children and adolescents is examined in a variety of ways. Some authors use data reported by parents ${ }^{(20)}$, while others have argued that self-reported data are accurate enough to measure food intake and food frequencies in children ${ }^{(21)}$. The cognitive abilities required to report food intake and food frequencies start to develop rapidly from the age of 8 years onwards. Cognitive processes become similar to those of adults at around the age of 10 years ${ }^{(22,23)}$. In the current study self-reports were used. There may be some misspecification of certain variables due to the unwillingness or inability of some participants to answer particular questions. The fact that $2 \cdot 3 \%$ of those claiming to eat all meals in a family setting also claimed to have skipped meals in order to watch television might be an indicator of the extent to which some answers were inaccurate, but it is also possible that these participants either ate with their family or skipped a meal - while others, who said they did not eat all meals with their family, simply ate alone. Validation of reliable measurement methods will be an important task for those who plan to do more research in this area.

Finally, it has to be noted that the confidence intervals for the family meal measure in Table 1 are exceedingly wide. The odds ratios for this variable are therefore probably unreliable approximations of the underlying relationship. The wider the confidence interval, the wider the range of values the odds ratio may have in the population from which our sample was drawn.

\section{Conclusions}

Adolescents who watched television during meals were more likely to skip meals in order to watch television. The same applied to computer games and reading books: use of those media during meals coincided with a much higher probability of meal skipping to use those same medium. In the case of television, the medium for which meal skipping was most prevalent, meal skipping was less likely as the adolescents ate their meals in a family setting more regularly. This suggests that using media during meals may encourage media meal skipping, while some protective or preventive effect may result from eating as many meals as possible as a family. The latter may only hold for television.

\section{Acknowledgements}

This research received no specific grant from any funding agency in the public, commercial or not-for-profit sectors. The authors report no conflict of interest. Both authors cooperated equally on all aspects of the study: study design, data gathering, statistical analyses, literature review, and discussion of the limitations and the results. The authors wish to acknowledge the enthusiasm and cooperation of the students, who received course credits for taking part in the data gathering process.

\section{References}

1. Rampersaud GC, Pereira MA, Girard BL, Adams J \& Metzl JD (2005) Breakfast habits, nutritional status, body weight, and academic performance in children and adolescents. J Am Diet Assoc 105, 743-760.

2. Mota J, Fidalgo F, Silva R, Ribeiro JC, Santos R, Carvalho J \& Santos MP (2008) Relationships between physical activity, obesity and meal frequency in adolescents. Ann Hum Biol 35, $1-10$.

3. Chapelot D, Marmonier C, Aubert R, Allègre C, Gausseres N, Fantino M \& Louis-Sylvestre J (2006) Consequence of omitting or adding a meal in man on body composition, food intake and metabolism. Obesity (Silver Spring) 14, 215-227.

4. Toschke AM, Küchenhoff H, Koletzko B \& von Kries R (2005) Meal frequency and childhood obesity. Obes Res 11, $1932-1938$.

5. Dubois L, Girard M, Potvin Kent M, Farmer A \& TatoneTokuda F (2009) Breakfast skipping is associated with differences in meal patterns, macronutrient intake and overweight among pre-school children. Public Health Nutr 12, 19-28.

6. Van den Bulck J \& Eggermont S (2006) Media use as a reason for meal skipping and fast eating in secondary school children. J Hum Nutr Diet 19, 1-10.

7. Coon KA, Goldberg J, Rogers BL \& Tucker KL (2001) Relationships between use of television during meals and children's food consumption patterns. Pediatrics 107, E7.

8. Higgs S \& Woodward M (2009) Television watching during lunch increases afternoon snack intake of young women. Appetite 52, 39-43.

9. Feldman S, Eisenberg ME, Neumark-Sztainer D \& Story M (2007) Associations between watching TV during family meals and dietary intake among adolescents. J Nutr Educ Behav 39, 257-263.

10. Stroebele N \& de Castro JM (2003) Television viewing is associated with an increase in meal frequency in humans. Appetite 42, 111-113.

11. Eggermont S \& Van den Bulck J (2006) Nodding off or switching off? The use of popular media as a sleep aid in secondary school children. J Paediatr Child Health 42, 428-433.

12. Neumark-Sztainer D, Hannan PJ, Story M, Croll J \& Perry C (2003) Family meal patterns: associations with sociodemographic characteristics and improved dietary intake among adolescents. J Am Diet Assoc 103, 317-322. 
13. Larson NI, Neumark-Sztainer D, Hannan PJ \& Story M (2007) Family meals during adolescence are associated with higher diet quality and healthful meal patterns during young adulthood. J Am Diet Assoc 107, 1502-1510.

14. Videon TM \& Manning CK (2003) Influences on adolescent eating patterns: the importance of family meals. J Adolesc Health 32, 365-373.

15. Borgatta EF \& Bohrnstedt GW (1981) Level of measurement: once over again. In Social Measurement: Current Issues, pp. 23-27 [GW Bohrnstedt and EF Borgatta, editors]. Beverly Hills, CA: Sage.

16. Gable S, Chang Y \& Krull JL (2007) Television watching and frequency of family meals are predictive of overweight onset and persistence in a national sample of school-aged children. J Am Diet Assoc 107, 53-61.

17. Haapalahti M, Mykkänen H, Tikkanen S \& Kokkonen J (2003) Meal patterns and food use in 10- to 11-year-old Finnish children. Public Health Nutr 6, 365-370.
18. Fabritius K \& Rasmussen M (2008) Breakfast habits and overweight in Danish schoolchildren. The role of socioeconomic positions. Ugeskr Laeger 170, 2559-2563.

19. MacFarlane A, Crawford D, Ball K, Savige G \& Worsley A (2007) Adolescent home food environments and socioeconomic position. Asia Pac J Clin Nutr 16, 748-756.

20. Braet C \& Van Strien T (1997) Assessment of emotional, externally induced and restrained eating behaviour in nine to twelve-year-old obese and non-obese children. Behav Res Ther 35, 863-873.

21. Rockett HR \& Colditz GA (1997) Assessing diets of children and adolescents. Am J Clin Nutr 65, 4 Suppl., 1116S-1122S.

22. Livingstone MB \& Robson PJ (2000) Measurement of dietary intake in children. Proc Nutr Soc 59, 279-293.

23. Baranowski T \& Domel SB (1994) A cognitive model of children's reporting of food intake. Am J Clin Nutr 59, 1 Suppl., 212S-221S. 